\title{
Die Image-Lüge in der Massendemokratie - über einen neuen Typ der politischen Lüge
}

Le mensonge lié à l'image dans la démocratie de masse - à propos d'un nouveau type de mensonge politique

Lies to keep public images polished - On a recent type of political lie in mass democracy

\section{Clemens Knobloch}

\section{(2) OpenEdition}

\section{Journals}

Édition électronique

URL : http://journals.openedition.org/ceg/1497

DOI : $10.4000 /$ ceg. 1497

ISSN : 2605-8359

\section{Éditeur}

Presses Universitaires de Provence

Édition imprimée

Date de publication : 15 juin 2015

Pagination : 131-146

ISBN : 978-2-85399-993-9

ISSN : 0751-4239

\section{Référence électronique}

Clemens Knobloch, « Die Image-Lüge in der Massendemokratie - über einen neuen Typ der politischen Lüge », Cahiers d'Études Germaniques [Online], 68 | 2015, Online erschienen am: 17 Dezember 2017, abgerufen am 01 Dezember 2020. URL : http://journals.openedition.org/ceg/1497 ; DOI : https://doi.org/10.4000/ceg.1497 


\section{Die Image-Lüge in der Massendemokratie - über einen neuen Typ der politischen Lüge \\ Clemens KNOBLOCH}

Universität Siegen

In The Bonfire of the Vanities, Tom Wolfes New-York-Roman aus den 80er Jahren des vorigen Jahrhunderts, verfährt sich ein früher Vertreter der Finanzzockerspezies mit seiner Geliebten und seinem 50.000 \$-Mercedes in der schwärzesten Bronx. Dort wird der Wagen auf einer Rampe durch ein Hindernis gestoppt, zwei schwarze Jugendliche tauchen auf und bieten Hilfe an. Bei der panischen Flucht des Pärchens, das fürchtet, Opfer eines Überfalls zu werden, wird einer der Jugendlichen durch den startenden Wagen erfasst und verletzt und fällt am folgenden Tag im Krankenhaus ins Koma. Der Rest des Romans erzählt, wie eine Vielzahl weiterer Akteure diesen „Fall“" okkasionalistisch so umbiegt, dass er maximalen Nutzen und Gewinn für das je eigene Image abzuwerfen verspricht: Peter Fallow, der bis dahin erfolglose und versoffene Sensationsjournalist hat endlich seinen scoop und kommt groß heraus. Reverend Bacon, umtriebiger Wortführer aller New Yorker Minderheiten (der Schwarzen, der Frauen, der Schwulen), baut mit seiner Hilfe das (zufällige und farblose) Opfer der hit-and-run-Affäre zu einer Lichtgestalt auf und organisiert Demonstrationen gegen die weiße Klassen- und Rassenjustiz. Abe Weiss, District Attorney im Wahlkampf, hat endlich den spektakulären Fall, der seine Wiederwahl in der schwarzen Bronx sichern wird, wenn er sich nur erfolgreich als harter Verfolger auch eines weißen Wall-Street-Millionärs inszeniert. In diesem literarischen Labor bilden Ereignisse den Rohstoff, aus dem die beteiligten Akteure imagekonforme und ergo für die Eigengruppe plausible Narrative formen. Es sind diese Images, die öffentlich aufeinander treffen. Das wirkliche Opfer interessiert niemanden und der vermeintliche Täter, schuldig bestenfalls des Ehebruchs, wird zwischen ihnen zermahlen.

Ob solche imagekonformen Transkriptionen als „Lüge“ angesprochen werden können, bleibt zu prüfen. Fest steht jedoch zweierlei: Die von Tom Wolfe literarisch aufgespießte Praxis imagekonformer Transposition dominiert das öffentliche Sprechen unter den Bedingungen einer massenmedialen Aufmerksamkeitsökonomie. Und diese Praxis ist konstitutiv für die spezifische Form, die „Lüge“ als Teilnehmerkategorie in derartigen Settings annimmt. Hannah Arendt (1972) hat die Risiken und Nebenwirkungen solcher Image-Lügen erstmals herausgearbeitet: Sie erzeugen dynamische Konsistenzzwänge, in denen die politischen Akteure sich leicht verrennen, und sie binden die Eigengruppe, während der jeweilige Feind 
oder Gegner durch sie nie getäuscht wird. Und sie sind nicht eigentlich durch Tatsachenwahrheiten widerlegbar, sie können bestenfalls zusammenbrechen und geben dann den Blick frei auf „Tatsachen“, die bislang von ihnen verdunkelt oder verstellt waren. Wo die Image-Lüge dominiert, gibt es für das öffentliche Sprechen zwar Dummheiten oder Themen, die gemieden werden müssen (im deutschen Bundestagswahlkampf 2013 griffen sich die Zeitungskommentatoren an den Kopf, weil Peer Steinbrück den deutschen Bundeskanzler für unterbezahlt erklärte, wo er doch das Image des Kämpfers für mehr soziale Gerechtigkeit so dringend brauchte!), aber die „Lüge“ gibt es dann nur im Bereich persönlicher Moral und Justiziabilität. Die moralisch eskalationsträchtigen Image-Lügen, mit denen die modernen Kriege begonnen wurden (von serbischen Gräueltaten im Kosovo bis zu irakischen Massenvernichtungswaffen), haben auch nach ihrer Aufdeckung keinen Politiker zu Fall gebracht. Als (dann gefährliche) Teilnehmerkategorie fungiert die Lüge nur bei persönlicher Vorteilnahme - das signalisiert dem Publikum zugleich Nähe und Distanz, indem Politiker auf den eigenen Vorteil aus sind (wie wir), dabei aber (anders als wir) wirklich viel riskieren. Man kann sie also aus dem sicheren Feld der „Normalität“ heraus zugleich heimlich bewundern und doch froh sein, dass man selbst (in der „Mitte“) vor derartig gefährlicher medialer Aufmerksamkeit geschützt ist.

„Images“ sind nicht darauf angelegt, wahr zu sein oder vollständig mit den Akteuren übereinzustimmen. Sie müssen konsistent und zustimmungsfähig sein und sie erzeugen ein Plausibilitätsfeld, außerhalb dessen Kommunikation teuer und riskant wird. Insofern beeinflussen sie den „Preis“ der Lüge ebenso wie den „Preis“ der Wahrheit.

Ich möchte versuchen, den dynamischen Konsistenzzwängen öffentlicher ImageLügen an einigen Beispielen nachzugehen, die es erlauben, die Bindewirkungen solcher Konstellationen auf die Eigengruppe schärfer von den Außenwirkungen abzusetzen - mit Blick auf die Frage, welche Folgen das in beiden Sektionen für die Teilnehmerkategorie „Lüge“ hat.

\section{Theoretische Annahmen}

Zunächst gilt für alle diskurslinguistischen Untersuchungen der „Lüge“, dass man sich im Schatten Foucaults von Problemen der Aufrichtigkeit einzelner Sprecher, der strategischen Manipulation des Publikums etc. ebenso abgekoppelt hat wie vom sprachkritischen Topos der selbst im Kern lügenhaften Sprache. Stattdessen geht es um die historisch variable Eigenlogik der öffentlichen Macht-Wissen-RedeKomplexe, in denen ganz praktisch verhandelt wird, was überhaupt zugelassen werden soll auf dem politischen Kampfplatz konkurrierender Wahrheiten.

Vorerst gibt es wenig Grund, sich in den vor allem im populären Neoevolutionismus der jüngsten Zeit geführten Debatten über die anthropologisch (oder sonst wie) naturalisierte Lüge zu positionieren (vgl. Sommer 1993). Tendenziell sucht die genegoistische Fraktion Täuschung, Übervorteilung und Lüge in allen Formen des Signalverkehrs als default zu etablieren, eben des unweigerlichen Drangs der Gene, 
ihre eigene Proliferation zu maximieren. Ausnahmen bilden in der Hauptsache die sogenannten „teuren Signale“, die darum weitgehend fälschungssicher sind, weil für den Signalgeber viel auf dem Spiel steht, weil er keine Kosten scheut oder hohe Risiken eingeht (Zahavi \& Zahavi 1998). Auf der anderen Seite steht die klappsymmetrische Annahme von der „natürlichen“ Kooperationsbereitschaft der Menschen, die dann kulturell konditioniert und dergestalt gebahnt wird, dass überhaupt eine Normierung axiomatisch sinnvoll wird. Wenn wir „von Natur" lügen, ist eh alle Moral vergebliche Liebesmüh, und wenn wir gar nicht anders können als kooperativ und aufrichtig sein, dann sind Lügner krank, deviant, Irrläufer der Evolution:

\footnotetext{
Das freizügige Teilen von Informationen scheint etwas zu sein, das schon bei sehr kleinen Kindern auf natürliche Weise vorkommt. Zwar lernen Kinder auch sehr bald zu lügen, aber dies tritt erst einige Jahre später auf und setzt vorheriges Vertrauen und Kooperationsbereitschaft voraus. Wenn die Menschen nicht davon ausgingen, dass sie sich auf die Hilfsbereitschaft anderer verlassen könnten, würden Lügen gar nicht erst funktionieren. (Tomasello 2010: 31)
}

Im Allgemeinen etablieren sich normative Ordnungen, die offenkundige Lügen gegenüber der Eigengruppe stark sanktionieren, während die Beurteilung von Lügen gegenüber Fremdgruppen von Nachsicht bis zur taktischen Befürwortung und Entschuldigung changiert. Das ist eine krude Vereinfachung der Lage, sie dürfte jedoch ausreichen, um verständlich zu machen, was sich in der massendemokratischen Image-Lüge an dieser elementaren Konstellation verändert. Und das ist vor allem der Umstand, dass die „Aufrichtigkeit“ gegenüber der Eigengruppe zurücktritt gegenüber der kommunikativen Funktion der Bildung und Festigung moralischer Gemeinschaften, die als solche nach außen, gegenüber den (ständigen oder fallweise improvisierten) Fremdgruppen inszeniert werden. Anders gesagt: Man täuscht die Eigengruppe, um sie als moralische Gemeinschaft gegenüber den Fremdgruppen zu stärken, die man ohnehin in der Sache weder täuschen noch überzeugen kann, aber auf der Ebene der Gemeinschaftsbildung moralisch ausstechen möchte. Wie Hannah Arendt (1972) notiert, hat die politische Image-Lüge ohnehin keine Chance, den Gegner zu täuschen oder zu blenden. Die Image-Lüge entfaltet Druck vor allem innerhalb der Eigengruppe, sie hemmt und bindet diejenigen, die auch innerhalb der Eigengruppe „darauf bestehen, von Tatbeständen und Geschehnissen zu sprechen, die dem image nicht entsprechen“ (Arendt 1972: 81).

Erkennbar unterstellt Hannah Arendts scharfsichtige Analyse der Image-Lüge als politischen Hintergrund die Konstellation des Kalten Krieges. Ihr exemplum ist der Vietnamkrieg, weshalb für sie vor allem die Konsequenz in den Vordergrund tritt, die darin besteht, dass als Repräsentant des äußeren Feindes wahrgenommen wird, wer innerhalb der Eigengruppe den Tatsachen zu ihrem Recht verhelfen will (und damit das Image stört). Diese Konsequenz ist mit dem Ende des Kalten Krieges nicht völlig verschwunden, sie tritt aber angesichts der multiplen, asymmetrischen und von Fall zu Fall improvisierten „Feindlagen“ in den Hintergrund. In Reinform findet man sie noch gegenüber dem Bedrohungs- und Feindbild des ,internationalen Terrorismus“, aber angesichts der qua „Terrorismusgefahr" gerechtfertigten geheimdienstlichen Totalüberwachung von allem und jedem („befreundete“ Politiker 
eingeschlossen) werden die Kollateralschäden der Image-Lüge auch an dieser Front unübersehbar. Was nämlich den „eigenen“ Laden qua Image zusammenhalten soll, das kann ihn auch in die Luft sprengen. Und wenn sich diejenigen, die noch eben eigene Überwachungsmaßnahmen gegenüber der Öffentlichkeit mit der „Terrorismusgefahr“ legitimiert haben, mit einem Male selbst als (aus eben diesem Grunde) Überwachte wieder finden, dann bedeutet das zumindest einen Image-GAU.

Umgekehrt belegen die „Fälle“ Manning und Snowden, dass die Weltmacht USA die öffentliche Kommunikation von Tatsachenwahrheiten wie ein Schwerverbrechen verfolgt. Was Hannah Arendt vor vierzig Jahren schrieb, wirkt heute, als sei es just für diese Fälle formuliert:

Wo prinzipiell und nicht nur gelegentlich gelogen wird, hat derjenige, der einfach sagt, was ist, bereits zu handeln angefangen, auch wenn er dies gar nicht beabsichtigte. (Arendt 1972: 75)

Während gewöhnliche Tatsachenwahrheiten, ihrer Kontingenz wegen, politisch neutral sind, wird im Falle der systematischen politischen Lüge (von der man bei geheimdienstlicher Tätigkeit getrost ausgehen kann) das einfache Aussprechen der Wahrheit zur (häufig nicht ungefährlichen) politischen Handlung.

Im Detail lassen sich da wunderbare Beobachtungen anführen: So hat, auf dem absoluten Höhepunkt der offiziellen (und parteiübergreifenden) Empörung über die geheimdienstliche Überwachung der Bundeskanzlerin deren Innenminister doch tatsächlich ganz unsensibel vorgeschlagen, die Datensammlung der LKW-Maut für die „Verbrechensbekämpfung“ den Behörden zugänglich zu machen. Es kommt aber natürlich gar nicht gut an, wenn die jammernden Überwachten just im Zenith des öffentlichen Verständnisses die Überwachung dieser Öffentlichkeit weiter verstärken wollen! Man kann nicht unbegrenzt überwachen und gleichzeitig die Empörung über die eigene Überwachung in Anspruch nehmen. Augenblicklich wird aber bei der politischen Klasse letztere benötigt. Weshalb auch der Innenminister diesen Vorschlag ganz rasch wieder abgeräumt (bekommen) hat. Würde Deutschland aber erst einmal in den exklusiven Club der globalen geheimdienstlichen Überwacher aufgenommen, wäre der Imageschaden partiell geheilt.

Eine weitere Komplikation gegenüber der oben skizzierten Ausgangskonstellation besteht im vielfältigen Wechsel der fallweise adressierten Freund-undFeindkollektive. Wer nach der einen Ratio zum „Wir“ gehört und durch das kommunizierte Image gebunden werden soll, der kann bereits im nächsten Augenblick (oder mit Bezug auf das nächste Thema etc.) zur ohnehin nicht überzeugbaren Außengruppe gehören. Wir-Identitäten - auch das ein soziologischer Gemeinplatz - sind multipel und müssen von Fall zu Fall neu improvisiert werden, was beständig Friktionen und Widersprüche erzeugt, die zu Nachfragen führen und bearbeitet werden müssen. Das gilt auch für die zugehörigen images.

Vom jeweiligen Image her entwickelt sich eine Art streamlining des Sagbarkeitsraumes. Images erzeugen kommunikative Konsonanz- und Konsistenzzwänge, die stets berücksichtigt werden wollen. Kognitiv und emotional/ evaluativ dissonante Tatsachen und Meinungen werden nicht unwahr, sie bedürfen zunächst alternativer Deutungen oder alternativer Fassungen oder sie müssen ganz gemieden werden (wie das Thema doping im Fußball). Die Sprecher bewegen 
sich im Sagbarkeitsraum ihrer gepflegten Images, in der Sphäre der Darstellung. Gefahr droht ständig aus der Sphäre der Herstellung, für die auf der Hinterbühne des politischen Geschehens eine ganz andere Logik gilt: die Logik der Macht, in der Kompromisse, Zugeständnisse, Bündnisse mit den Inhabern von Sanktionspotential etc. gemacht werden müssen. Für den politischen Gegner ist darum das gesamte Image die Lüge, welche die ,wirkliche“" Machtlogik der Hinterbühne verdeckt. Für die Eigengruppe, solange sie jedenfalls auf dem vereinbarten Image mitzusegeln gedenkt, kann vorerst nur das als „Lüge“ gelten, was das Image der Eigengruppe zu beschädigen droht, und das ist entweder ein potentiell image-dissonantes Ereignis, ein imageinkongruenter Akteur oder eine seiner Handlungen, die nach außen sichtbar macht, dass die Person ,hinter“ dem Image eigene, womöglich moralisch anstößige oder der Eigengruppe schädliche Ziele verfolgt.

Immer wieder sind es unliebsame Tatsachen und Ereignisse, die die Konsistenz kommunizierter Images bedrohen. Und der Druck, sich in das riskante Feld der wirklichen Lüge (d.i. des Abstreitens einer Tatsache oder des Behauptens einer Nichttatsache) zu begeben, wächst vor allem dann, wenn Imagekonsistenz oder Imagekongruenz auf dem Spiel stehen. Und zwar ganz einfach darum, weil in dieser Konstellation der Preis der Wahrheit deutlich höher wird als der Preis der Lüge. Das jedenfalls ist meine These, die der folgende „Fall“ illustriert.

\section{Fall 1}

Übersichtlich vereint sind alle bestimmenden Faktoren der Image-Lüge vom sauberen Hochleistungssport. Ganz unterschiedliche Wir-Gruppen leben gut von diesem Image und haben ein ebenso lebhaftes wie gemeinsames Interesse an seiner Erhaltung:

[a] Die Werbeträger, die von der Beliebtheit und Aufmerksamkeitsprominenz des Leistungssports gleich mehrfach zehren. Sie vermarkten die eigenen Waren vermittels der öffentlichen Aufmerksamkeit und sakralisieren sie zugleich mit der Prominenz der Sport-Heroen.

[b] Die Politiker, die sich gerne im Ruhm der Sportprominenz sonnen und zugleich der Öffentlichkeit übermitteln, dass sie - ganz wie das Publikum - Fans der populären Helden sind.

[c] Noch einmal die Politiker, welche die beträchtlichen kollektivsymbolischen Ressourcen des Leistungssports bei jedem geeigneten Anlass für ihre Zwecke einsetzen: Sport ist „die bessere Gesellschaft“, wo Team- und Einzelleistung zählen, wo der Bessere gewinnt, wo jeder eine Chance hat, wo der Trainer gefeuert wird, wenn er's nicht bringt, wo sich alle gesellschaftlichen Schichten treffen, wo der Nationalismus ohne schlechtes Gewissen von der Kette gelassen werden kann etc. Die kollektivsymbolische Bedeutung des Leistungssports besteht darin, dass er ein „hyperrealistisches“" und leicht verständliches Projektionsfeld für die demonstrativen Ideale des modernen Kapitalismus liefert. Und wenn sich herausstellt, dass auch die „bessere“ Welt des Sports total vermachtet, mafiös, korrupt und geldgesteuert 
ist, dann mindert das eine ansonsten höchst ergiebige Quelle von Ressourcen symbolischer Macht. ${ }^{1}$

[d] Die großen Sportveranstalter und Sportverbände bis hin zum IOC, die nicht nur in ihrer Gesamtheit ein korruptionsaffiner und kriminalitätsnaher Zweig des Ganz Großen Geldes sind, wie man nicht erst seit Hoeneß, Katar und der Nürburgring-Affäre weiß, die darüber hinaus (der Interessenüberschneidung wegen) auch privilegierten Zugang zu öffentlichen Steuerkassen haben.

[e] Und schließlich die Leistungssportler selbst, die, trotz hoher und höchster Einkommen, diejenigen sind, welche das schwächste Glied in dieser Imageverkettung bilden, weil auf ihnen die ganze Last dieser kollektiven Imagefiktionen lastet. Sie sind es, die gekreuzigt werden, wenn die ganze Sache zusammenbricht. Sie sehen am Ende so aus, als seien sie, durch Geld, Ruhm und Ehre geködert, auf die schiefe Bahn geraten, während die anderen Profiteure dieses Systems in Ruhe weiterziehen können, weil man von ihnen nichts anderes erwartet, als dass sie ihre Geschäfte um jeden Preis betreiben. Wirklich verletzlich ist allein das Image der Sportler selbst, weil der Sport in der Massendemokratie moralisch und symbolisch als die „,bessere“ Projektion der Gesellschaft kodiert ist. Auch „Schurkenstaaten“ nutzen darum gerne das Image internationaler sportlicher Großveranstaltungen, um sich dem Rest der Welt in positivem Licht zeigen zu können. Sie wissen, dass die Sportler, um ihr eigenes Image zu erhalten, auch aus den Zwängen der übergreifenden Imagefiktion nicht aussteigen können.

Wenn etwas herauskommt über weit verbreitete „unsaubere“ Praktiken in einer Leistungssportart, dann kommt es zum Skandal, obwohl man weiß, dass sich vielerlei dubiose Interessen (Macht- wie Geldinteressen) im Leistungssport tummeln und kreuzen. Obwohl? - Nein, weil man das weiß, denn auch diese Eigenschaften medialer Skandale und Image-Lügen sind im Leistungssport rein ausgeprägt: Der Skandal macht nur aktenkundig und beweisbar, was ohnehin jeder schon weiß, der seine Murmeln beieinander hat. Schließlich muss die Skandalisierung an ein Deutungsmuster andocken, das im Publikum weit verbreitet und tief verankert ist in diesem Falle an das Deutungsmuster, wonach im „Oben“ der Gesellschaft nicht nur die Leistungseliten, sondern auch die egoistischen, gierigen und verlogenen Schufte zu Hause sind.

Ein nicht uninteressantes und ebenfalls imagegetriebenes Muster kann man bei den großen Sportverbänden studieren, sobald sich ein Skandal am Horizont abzeichnet, sei es die staatlich ermutigte und geförderte Dopingpraxis auch in Westdeutschland oder seien es die Zustände auf den Weltmeisterschaftsbaustellen in Katar: Wenn die (ebenso gefährlichen wie gefährdeten) Tatsachen nicht mehr unter der Decke gehalten werden können, schlagen sich die Verbände umstandslos und von einem Augenblick zum nächsten auf die Seite der brutalstmöglichen Aufklärung des Skandals. ${ }^{2}$ Wer sein sauberes Image nicht mehr retten kann, der etabliert wenigstens

\footnotetext{
1 Weswegen auch alle Alarmglocken zu läuten beginnen, wenn „die Massen“ (wie jüngst in Brasilien) gegen die Verschwendung öffentlicher Mittel für prestigeträchtige sportliche Großveranstaltungen auf die Straße gehen.

2 Ein Muster, das natürlich auch anderweitig in der politischen Kommunikation zu beobachten ist, prominent im CDU-Parteispendenskandal der späten Kohlära.
} 
das Ersatzimage der Aufklärung - und nutzt dieses neue Image alsdann, um imageschädliche Tatsachen auch weiterhin nach Kräften unter der Decke zu halten.

Mit wachsenden Spannungen zwischen den verschiedenen Handlungsfeldern, die das Kollektivsymbol Leistungssport ausbeuten, wird die Rolle attraktiver, die neudeutsch als die des whistleblowers bezeichnet wird. Das ist die Person, die ihr Insiderwissen über die höchst imagedissonanten Tatsachen des Feldes der Öffentlichkeit und/oder den Medien zur Verfügung stellt, um den Preis, augenblicklich von allen Imagegemeinschaften verstoßen $\mathrm{zu}$ werden, die davon profitieren, dass die Tatsachen jedenfalls nicht aktenkundig werden (gerade weil jeder sie ahnt). Individuelle Beichten hingegen, wie wir sie (vielfach als großes Theater!) von Dopingsündern medial aufgetischt bekommen, sind dagegen aus leicht verständlichen Gründen nicht nur beim Publikum beliebt, sondern auch bei den Profiteuren der kollektiven Imagefiktion. Sünder, die medienwirksam beichten, übertragen nicht nur ihren sportlichen Ruhm in eine moraloide Währung, sie polieren auch das Gesamtimage vom sauberen (oder jedenfalls der Selbstreinigung fähigen) Sport. All das verdampft augenblicklich, wenn jemand aufsteht und sagt: Was der „Sünder“ da beichtet, ist exakt das, was alle getan haben und tun.

\section{Fall 2}

Auf eine ausgesprochen harte Probe werden die Tatsachen stets dann gestellt, wenn ein medial hoch moralisiertes Ereignis ins öffentliche Gedächtnis rückt, das drastisch unterstreicht, was jeder weiß. Die 350 im Herbst 2013 vor Lampedusa im Mittelmeer ertrunkenen afrikanischen Flüchtlinge haben durch ihre schiere Zahl kurzzeitig ins öffentliche Bewusstsein gerückt, was zwischen den afrikanischen und den südeuropäischen Küsten ständig passiert. Solche Ereignisse stellen hohe Anforderungen an alle Beteiligten, so sie ihr Image erfolgreich managen wollen. Nicht dass die Tatsache des massenhaften Flüchtlingstodes von irgendjemandem geleugnet würde, wohl aber, dass die europäischen Behörden diesen Tod nach Kräften befördern, weil sie potentielle Flüchtlinge abschrecken und entmutigen wollen. So hallte zwar das große Wort von der „Schande“ durch die Medien, gleichzeitig war jedoch im Kleingedruckten zu lesen, dass niemand auch nur daran dachte, das unter Berlusconi verabschiedete italienische Gesetz in Frage zu stellen, das jeden, der einem in Seenot geratenen Flüchtlingsboot hilft, mit einer Anklage wegen Beihilfe zum Menschenhandel bedroht. Auch ein halbherziger Versuch, per Erlass die Frontex-Schiffe zur Seenothilfe zu verpflichten, scheiterte am Einspruch der Betroffenen. Weil aber wenigstens der öffentliche Anschein von Tätigkeit erweckt werden musste, spendierte man rasch 350 Millionen Euro an Eurosur für die technische Perfektionierung der Küstenüberwachung in Afrika. So hat man immerhin das Kunststück geschafft, einen moralischen Skandal als Anlass zur weiteren Verschärfung der Lage einzusetzen.

In dieser Konstellation gab (und gibt) es hoch widersprüchliche Signalanforderungen, und dem entsprechend drohen Imagekollisionen. Der mediale moralische Druck schafft eine „Es-muss-etwas-geschehen“-Lage, zugleich zieht er 
den innereuropäischen Konflikt ans Tageslicht, in dem die Mittelmeerländer auf den Norden zeigen und sich demonstrativ mit dem Flüchtlingsproblem „,allein gelassen“ fühlen, während der Norden im Gegenzug erinnert, dass er viel mehr Flüchtlinge aufnimmt als die Mittelmeerländer selbst. Zugleich treiben im Windschatten des moralischen Skandals diejenigen ihre Sache weiter, die den Umgang mit Migration und Flucht „normalisieren“ und Einwanderung an ein (jederzeit den ökonomischen Erfordernissen anpassbares) Punkteregime koppeln wollen - und die (wahrscheinlich zu recht) davon ausgehen, dass die schiere Möglichkeit einer legalen Einwanderung (z.B. für „Qualifizierte“ oder Altenpfleger) das Geschäft der Menschenhändler dämpfen würde. Über all diesen konfligierenden Image-Anforderungen thront dann noch der dissonante Druck, allen „Fluchtbereiten“ in Nordafrika und anderswo die unbedingte Abwehrbereitschaft der EU-Staaten unmissverständlich zu signalisieren, weil alles andere als „Ermunterung“ zur Flucht ausgelegt werden könnte.

Es handelt sich um eine Lage, in der zahlreiche widersprüchliche ImageKomponenten gleichzeitig optimiert werden müssen, mit der Folge, dass jede halbwegs bestimmte Aktion immer einen großen Teil des Publikums verärgern muss. Darum verschwinden solche Themen schnell wieder von der medialen Agenda. Bis zum nächsten Skandal.

Nun soll es hier aber nicht um Imagepolitik im Allgemeinen, sondern um die Image-Lüge gehen. Frank Schirrmacher (2013) hat in seinem kulturkritischen Essay auf die „erzieherischen“ Wirkungen spieltheoretischer Optimierungspraktiken hingewiesen, wie sie auf den Finanzmärkten, im politischen Geschäft und in anderen Sphären strategischen Handelns anzutreffen sind: Jeder ist auf eigene Faust ein Narr, wenn er nicht als default damit rechnet, dass sein Gegenüber in geschäftsähnlichen Beziehungen (und welche wären das nicht?) als strategischer Nutzenmaximierer agiert und nicht als ehrlicher Makler. Und vor diesem Hintergrund muss der „Preis“ der Image-Lüge kalkuliert werden. Wenn nun die Pflege und Erhaltung des jeweiligen Images zur zentralen Ressource der Meinungsmacht avanciert, dann ist es der aktuelle Kurswert dieser Ressource, der das Verhältnis zu den „Tatsachenwahrheiten“ bestimmt. Wer das globale Image des unerbittlichen Anführers im „Krieg gegen den Terror" pflegt, der wird auf dem Feld der globalen Überwachung zugeben, was sich nicht mehr abstreiten lässt und alles für gerechtfertigt im Rahmen der eigenen Mission erklären. Dieser Komplex definiert dann potentiell dissonante Tatsachen: z.B. wenn im Rahmen dieser „Mission“ Industriespionage betrieben wird, wenn „befreundete“ Staatsoberhäupter abgehört werden, wenn die Geheimdienste so viel Hinterbühnenwissen ansammeln, dass sie alle Akteure erpressen und jedes Image zerstören können und damit faktisch die Macht im Staate übernehmen etc. Jeder Sprecher kalkuliert den „Preis“, der für Tatsachenbehauptungen zu entrichten ist, vor dem Hintergrund der Imagefolgen, die zu gewärtigen sind.

In besonderem Maße angewiesen auf ein intaktes Image sind natürlich medienöffentliche Moralakteure. Deren Meinungsmacht hängt ganz und gar am aktuellen Wert des Images (während genuine Macht- und Wirtschaftsakteure auch über andere Ressourcen verfügen). 


\section{Fall 3}

Hier mag als prominentestes Beispiel die (imagemäßig arg gebeutelte) katholische Kirche dienen. Ein nüchterner Beobachter der Medienszene wird kaum abstreiten, dass es in allen die katholische Kirche betreffenden öffentlichen Angelegenheiten eine markante journalistische Jagdlust und Hämebereitschaft gibt, ausgelöst vermutlich durch die kaum zu übersehenden Dissonanzen zwischen moralischem Image und machiavellistischer Realität.

Der „Fall“ des Limburger Bischofs Tebartz von Elst ist inzwischen hinreichend durchgekaut. Womöglich ist die (eigentlich von der Elbphilharmonie bis zum Berliner Flughafen ganz normale) Bauverteuerung auch deshalb ins Visier der medialen Aufmerksamkeit geraten, weil sie in schreiendem Kontrast zu den rührenden Reparaturversuchen steht, die der neue Papst am kirchlichen Image vorzunehmen sucht: Er kommuniziert, er mache sein Bett selbst und sei als Bischof mit der Straßenbahn zur Arbeit gefahren, er sucht das legendenreiche arm-und-naturverbunden-Image des heiligen Franziskus per Namensgebung auf die Mühlen einer Kirche zu lenken, die in der jüngeren Vergangenheit eher durch Geldwäsche, Pädophilieskandale (nebst deren Vertuschung) und die innerkirchliche Rehabilitierung klerikalfaschistischer Antisemiten und Holocaustleugner in den Schlagzeilen war. Zwar glänzt die katholische Kirche auch durch ihre Bereitschaft, Sünden zu vergeben, aber alles, was den Strudel ihres Image-GAUs noch reißender werden lässt, muss selbstverständlich ohne größeres Aufsehen aus den Schlagzeilen geleitet werden Die Versuche des Bischofs, zunächst die Limburger Finanzaufsicht und die Vermögensverwaltung und sodann auch den Vatikan in die Schuld „einzubinden“, hätten auch jedem Politiker zur Ehre gereicht. Zusammen mit dem massiven Unmut, den die bischöfliche Herrschaftspraxis aber bereits an der katholischen Basis ausgelöst hatte, dürften diese Einbindungsversuche eher die Bereitschaft des Umfelds gestärkt haben, den Bischof fallen zu lassen (anstatt mit ihm zu fallen).

Fast heiter sind da die guten Ratschläge besorgter katholischer Experten, wie die Kirche die moralische Meinungsführerschaft auf dem Sektor der Nächstenliebe wieder erobern könne, ohne ihre Vermögenswerte anzugreifen. In der Süddeutschen Zeitung empfiehlt ein versierter Unternehmensberater (mit McKinsey-Vergangenheit; Mitschke-Collande 2013), die katholische Kirche möge demonstrativ auf einen Teil ihrer staatlichen Einkünfte verzichten. Dabei erfährt der Leser, was vermutlich auch die meisten Katholiken nicht wissen: Dass nämlich die Kirchen - über die Kirchensteuer hinaus - in Deutschland bis heute staatliche Restitutionszahlungen in Höhe von über 400 Millionen Euro jährlich zur Kompensation der (beiläufig 210 Jahre zurückliegenden) Enteignung der Kirchengüter im Jahr 1803 erhalten. Diese Summe - so meint der McKinsey-Ratgeber - solle die katholische Kirche in eine Stiftung geben und zusammen mit staatlichen Stellen demonstrativ für wohltätige Zwecke verausgaben. So könne sie die Lufthoheit auf dem Kampffeld der Moral und der Sozialpolitik zurück gewinnen und müsse dabei nicht einmal ans Eingemachte, dann jedenfalls, wenn die Bistümer ihre Vermögensverhältnisse schonungslos offen legen würden. In solchen Ratschlägen, die jedes Unternehmen 
in vergleichbarer Lage wohl dankbar annehmen würde, steckt das ganze Dilemma moralisch kodierter Gemeinschaftsimages: In einer bezüglich moralischer Ansprüche reichlich desillusionierten Öffentlichkeit wirken derartige strategische Empfehlungen wie professionelle Reparaturversuche am Image, die das Misstrauen eher schüren als besänftigen. Auch textlinguistisch ist das mit Händen zu greifen, wenn Mitschke-Collande (2013) die Kirche zunächst rügt, die verhalte sich „fast zynisch“, wenn sie mit dem Argument Auskunft über ihr Vermögen verweigere, dass schließlich auch ein Unternehmer nicht sein Privatvermögen veröffentliche. Der gute Rat, den der Unternehmensberater der Kirche sodann erteilt, ist aber nicht weniger „zynisch“, besteht er doch in klassisch-moraloider Imagepolitik: demonstrativ zugunsten eines ,guten“ Zwecks auf einen Anspruch zu verzichten, der ohnehin nicht mehr haltbar ist. Das als „Beitrag zur Entflechtung von Kirche und

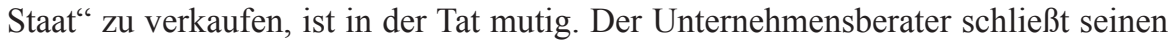
Rat mit dem Satz: „So müsste eine Institution agieren, die sich anders verhalten will als ein Unternehmen“. Es hieße wohl besser: die so aussehen möchte, als sei sie kein Unternehmen.

In der Massendemokratie ist das Image der Teilnehmer die entscheidende Schnittstelle für die symbolische Reproduktion von Zustimmung und Macht - natürlich nicht die der institutionellen Reproduktion von Macht, die über ganz andere Ressourcen und Logiken verfügt. Aber wie zu allen Zeiten dient die symbolische, nach außen sichtbare Seite der Reproduktion von Macht immer auch dazu, die Hinterbühne in ein offiziöses Halbdunkel zu hüllen, von dem der normale Teilnehmer immer nur ahnt, dass es da ziemlich hemdsärmelig zugehen muss. Die These ist: Schichten, Klassen, Weltanschauungsgruppen oder Milieus zerfließen als Basis für politische Macht und Zustimmung. Auch Themenlogiken lassen sich nicht mehr auf Dauer von einzelnen politischen Gruppierungen einhegen. Das Muster für die Nicht-Einhegbarkeit hoch resonanter Themen ist die Ökologie, deren Anliegen in rasantem Tempo von allen politischen Gruppierungen übernommen (und eo ipso verwässert) worden sind. Zustimmungsfähige Themen entstehen an den Rändern des politischen Geschehens, sorgen dafür, dass an diesen Rändern neue Kräfte und Gruppierungen aufkommen, die aber alsbald von den Mitte-Akteuren symbolisch enteignet werden, weil die deren Themen zugleich aufnehmen und entschärfen. Die einzige stabile Schnittstelle zwischen Akteur und Öffentlichkeit ist ergo das Image des Akteurs.

Die Image-Logik, von Hannah Arendt in ihrem klassischen Text von 1972 erstmals politisch seziert, breitet sich in der gesellschaftlichen und politischen Wirklichkeit aus. Sie hat in den letzten beiden Jahrzehnten z.B. das Bildungswesen erfasst, das imagemäßig zwischen „Exzellenz“ und „Hauptschule“ (Lasst alle Hoffnung fahren!) gespreizt ist. Der Typus der medialen Kontrolle, der auch öffentliche und staatliche Akteure unterworfen sind, legt es ihnen dringend nahe, in die Erhaltung eines positiven Images zu investieren.

Es geht jeweils um imagekonforme und wahrheitsfähige Versionen dessen, was passiert oder passiert ist, was getan wird oder getan worden ist. Die inhärente Logik des öffentlichen Verhaltens besteht darin, so lange wie irgend möglich an einem einmal übernommenen und aufgebauten Image festzuhalten, konträre 
Informationen möglichst wirkungsvoll zu dementieren oder aus der Öffentlichkeit zu halten, im Notfall und unter äußerstem Druck dann zu einem anderen positiv bewerteten Nebenimage zu wechseln. So wie das Bundesinstitut für Sportforschung augenblicklich (August 2013) die Flucht nach vorne antritt und versucht, das Image des Aufklärers auf die eigene Mühle zu lenken. Oder so wie das bayrische Justizministerium, das im Fall der Psychiatrisierung von Gustl Mollath so lange wie irgend möglich den Garant der Unfehlbarkeit der bayrischen Justiz gab, aber dann, als die Folgen sie einzuholen drohten, die Anweisung gegeben hat, den Fall gerichtlich neu aufzurollen, was wiederum den kommunikativen Übertritt zum Aufklärer-Image ermöglicht.

\section{Image und Lüge}

Nun sprechen wir hier über die Lüge, und Lüge ist ein hoch eskalationsträchtiger moralischer Vorwurf in der sozialen Nähekommunikation. Was aber ist eine Lüge in der medienöffentlichen Imagekommunikation der massendemokratischen Politik? Ist nicht jeder Versuch, ein konsistentes moralisches Erfolgsimage zu managen, schon per se eine Lüge? Oder wenigstens eine Aktivität, bei der die Unterscheidung zwischen Aufrichtigkeit und Lüge kaum Sinn ergibt? Und ist es nicht so, dass jeder das auch weiß?

Ich gebe ein extremes Beispiel aus der Werbebranche: Ein britischer Haferkeks (Patterson) wird mit einem Naturschutz-Image als ,orang utan friendly“ vermarktet. Ein treuherzig schauender rot-brauner Orang Utan ziert die Packung, und auf der heißt es, seit einigen Jahren würden keine Palmfette mehr in dieser Kekssorte verbacken, sondern nur noch Olivenöl, weil der Anbau von Ölpalmen den Lebensraum dieser putzigen (und uns so nahe stehenden) Kreaturen vernichte. So könne der Kunde durch den Verzehr dieser oat cake-Marke zum Schutz der Primaten beitragen. Solche „Moralisierung des Konsums“ gibt ein Modell der werblichen Imageproduktion, an der sich, wie Hannah Arendt festgestellt hat, auch die massendemokratische Politik orientiert. Sie führt zu einer symbolischen Verschmelzung (merger) von Anbieter und Konsument, die beide moralisch gehoben werden, der eine durch den demonstrativen Verzicht auf das umweltschädliche Palmfett, der andere durch seine moralisch angereicherte Konsumentscheidung. Der Image-Frame rahmt, adelt und verbindet alles, was in seinem Skopus geschieht. Aus einem banalen Haferkeks wird eine moralische Demonstration. ${ }^{3}$

Stellen wir uns nun vor, irgendeine Umweltschutzorganisation deckt auf, dass die Eigentümer der Keksfirma Patterson über undurchsichtige Kapitalverflechtungen der weltweit größte Betreiber von Palmölplantagen in Indonesien sei. Was wird passieren? Dazu gibt es zwei Thesen:

[a] Die These von der moralischen Fallhöhe, die umso größer wird, je penetranter das moralische Image ausfällt, das in der Medienöffentlichkeit getragen wird.

\footnotetext{
„A well rounded frame serves as an amplifying device. Since all aspects of living tend to become tied together by its symbolic bridges, each portion involves the whole." (Burke 1937: 103).
} 
Einschlägige Beispiele liefert etwa der Ansehensverlust, den medienöffentliche Moralagenturen erleiden, wenn sich herausstellt, dass hinter ihrer Fassade ganz andere Kräfte wirken. Ich denke an die katholische Kirche, die mit ihrer (im neoliberalen Konsumhedonismus atavistisch wirkenden) Sexualmoral natürlich durch den nachgewiesenen, aber lange vertuschten sexuellen Missbrauch von Kindern und Jugendlichen eine Art Image-GAU erlebt hat. Oder auch an die UmweltschutzNobelmarke World Wildlife Funds (WWF), von der zumindest behauptet wird, dass sie ihre Spendengelder auch einsetzt, um für ihre prominente und wohlhabende Kundschaft Großwildjagden und Edelurlaube in Regionen zu organisieren, deren indigene Bevölkerung zuvor aus „Naturschutzgründen“ umgesiedelt wurde. Das ist ein wenig so, als ob Greenpeace mit Spendengeldern eine Fracking-Firma betreiben würde, und exemplifiziert den öffentlichen Kollaps eines moralischen Images.

Es ist aber keineswegs ausgemacht, dass ein solcher Effekt auch dann eintreten müsste, wenn von den fraglichen Akteuren zwar ein moralisches Image, aber durchaus kein moralisches Handeln erwartet wird. Und das dürfte sowohl bei den oat-cake-Produzenten als auch bei massendemokratischen Politikern der Fall sein. Da könnte dann eher die Option [b] greifen:

[b] Die These von der aufgehobenen Selbstbindung für moralisch universalistische Imageproduktion, die etwa Neckel (2008) vertritt. Nach dieser Annahme verbreitet sich in der entfesselten Marktgesellschaft, in der jeder zugleich Erfolg haben und ein positives Image pflegen muss, quasi von selbst und automatisch die Gewissheit, dass jedes Image dem Erfolgszwang untergeordnet und daher für den Träger ohne Verbindlichkeit und Verpflichtungsgehalt sei:

\begin{abstract}
Das Eigenartige an der heute marktgängigen Angebotsmoral ist dabei, dass sie offenbar einem inneren Zwang jedweder moralischer Argumentation weitgehend entkommt: der Selbstbindung, der jeder unterliegt, der sich auf allgemeine Prinzipien beruft. Da der Universalismus ja in nichts anderem als im beanspruchten Recht auf das Eigeninteresse besteht, können die Segnungen des Egoismus die Moral nicht blamieren. Das klassische Muster der Moralkritik, Schein und Wirklichkeit miteinander zu konfrontieren, läuft ins Leere. (Neckel 2008: 38)
\end{abstract}

Wo strategisches streamlining aller nach außen preisgegebenen Informationen die normale, von allen erwartete Praxis ist, wird entweder alles zur Lüge - oder aber nur der komplette Zusammenbruch eines lange Zeit sorgfältig gepflegten Images. Und in beiden Fällen wird der Vorwurf der Lüge performativ völlig zahnlos. Welchen pragmatischen Sinn hat der Vorwurf, in Sachen doping gelogen zu haben, gegen einen ,erfolgreichen“ Radsportler, wenn doch seit langem völlig klar ist, dass jeder erfolgreiche Radsportler dopen muss, wenn er erfolgreich sein möchte? Auf das Branchenimage des Radsports ist dann jeder verpflichtet, der es in der Branche zu etwas bringen möchte. Die Lüge ist dann durchaus nicht individuell. Und wenn einzelne (dann natürlich die ganz besonders erfolgreichen!) herausgepickt und skandalisiert werden, dann dient das einerseits der Sensationslust des Publikums, das öffentliche Beichten und Abstürze schätzt: unnormale Fahrten durch die soziale Ordnung, zuerst (wie bei Sportlern und anderer massenkultureller Prominenz üblich) von unten nach oben, und dann wieder von oben nach unten. Andererseits aber handelt es sich auch um Bauernopfer, die es ermöglichen sollen, das Image der 
Branche insgesamt zu retten. Das geht nur, wenn moralisches Versagen, wenn die „Lüge“ individuell zugerechnet werden kann und eben nicht durch das offizielle Branchenimage eingefordert wird.

Mutatis mutandis gelten analoge Rahmenbedingungen auch für die massen- und mediendemokratische Politik: Wenn die strategische und konsistente Inszenierung eines Images von allen erwartet und Teil der allgemeinen Geschäftsgrundlage ist, dann verhält sich nur derjenige rational, der das mit Geschick tut und der gleichzeitig das Geschick und die Schlagfertigkeit aufbringt, seine Kontrahenten bei der Inszenierung ihres Images nachhaltig zu stören. Wettbewerb und Konkurrenz verlagern sich dann von ganz allein auf die Ebene der Image-Inszenierung, und bei einer Vorwahldebatte (wie beim „Fernsehduell“ zwischen Merkel und Steinbrück im Wahlkampf 2013) gilt derjenige als Sieger, der das eigene Image gewahrt und das der Konkurrenz gestört hat. Da Images per definitionem gar nicht darauf angelegt sind, „wahr“ zu sein oder mit den Akteuren, die sie vor sich her tragen, übereinzustimmen, erzeugt die Imageproduktion immer und zwangsläufig zugleich auch die Ressourcen, mittels deren Akteure zu Fall gebracht werden können, wenn sie sich zu weit vorgewagt haben. Handelt es sich jedoch um kollektiv bindende Gruppenimages, verlagert sich die Sanktionsmacht in die Eigengruppe, die den einzelnen opfert, um das Gruppenimage zu erhalten.

\section{Funktion konsistenter Images}

Wo die politischen Interessen- und Positionsunterschiede in der Sache geringfügig sind, wird die Image-Ebene umso wichtiger. In der sachlich weitgehend nivellierten massendemokratischen „Mitte“ bilden die politischen Akteure einerseits ein „Ensemble“, das ehrgeizige Macht-Aspiranten, die von den Rändern nachdrängen, gemeinsam neutralisieren und außen vor halten muss. Andererseits neigen die in den Hauptsachen einigen Akteure dazu, ihre unterschiedlichen Images kompensatorisch zu übertreiben, weil allein sie dem Publikum die Illusion einer echten Wahl vermitteln können. Daher hat der skeptische Beobachter der Szene manchmal den Eindruck, bei aller inszenierten Differenz steckten „die da oben“ doch im Grunde unter einer Decke.

Geht es um die Gunst der Wähler, dominiert das, was Erving Goffman als aggressive Verwendung der Techniken der Imagepflege bezeichnet (Goffman 1971: 30). Während die Akteure auf ein und derselben mikrosoziologischen Bühne gewöhnlich ein gewisses Maß an Kooperation vorführen und den andren dabei helfen, ihr Gesicht zu wahren (,Takt"), geht es beim politischen Wettkampf vor Publikum allein darum, das Image des anderen unglaubwürdig zu machen und das eigene zu polieren. Medientauglich und talentiert ist, wer den kleinsten Riss in der Fassade des anderen sofort entdeckt und einen Keil hineintreibt. In der Vorwahl-Runde war der SPD-Kandidat Steinbrück so unvorsichtig, sich auf das populistische Gebiet des Beamten-Bashing vorzuwagen. Die Zuwächse bei den Beamtenpensionen müssten beschränkt werden, sagte er. Worauf Merkel konterte, alle Polizisten, Feuerwehrleute und Lehrer sollten doch bitte genau hinhören, ausgerechnet der Sozialdemokrat Steinbrück (mit 1,3 Millionen Euro Vortragshonoraren und der zuvor bekundeten 
Ansicht, als Kanzler verdiene man viel zu wenig) wolle an ihre Altersbezüge. Es ist dabei wichtig festzuhalten, dass eine solche Episode nichts, aber auch wirklich gar nichts darüber aussagt, wer (und ob überhaupt) Beamtenpensionen kürzt.

Unter den Bedingungen globalisierter Medienkommunikation (auch das hat Hannah Arendt 1972 bereits festgehalten), ist es vor allem Motivation und Zusammenhalt der Eigengruppe, was durch spektakuläre Lügen über die moralischen Verfehlungen der Gegner gebunden werden soll. Besonders augenfällig wird das in den Kriegen der vergangenen Jahrzehnte. Sie beginnen alle mit einer die Eigengruppe „motivierenden“ und moralisch empörenden Schauergeschichte: die von Irakern angeblich verwüstete Neogeborenenstation eines Kuweiter Krankenhauses, serbische Gräueltaten im Kosovo, Massenvernichtungswaffen im Irak, und selbst der Krieg in Afghanistan folgte diesem Muster, denn mit dem Angriff auf das WTC 2001 hatte jedenfalls die damalige afghanische Regierung nicht das mindeste zu tun. Solche Lügen verändern die Handlungsbedingungen punktuell und lokal, es macht keinen Unterschied, ob sie später einmal als solche entlarvt werden. Ihren Dienst haben sie dann längst getan.

\section{Lüge, performativ}

Den praktischen Umgang der politischen Klasse mit dem Vorwurf der Lüge thematisiert ein FAZ-Kommentar („Nichts als die Wahrheit?“, FAZ vom 31.7.2013). Der Autor, Reinhard Müller, thematisiert das ganze Feld des Verschweigens, Täuschens, Tricksens, zunächst mit dem Tenor, dass naturgemäß niemand immer die volle Wahrheit sage, die ganze Wahrheit könne nämlich kein Mensch dauerhaft ertragen! Ganz ohne Lüge gehe es darum weder in der Politik noch im täglichen Leben. So weit, so trivial. Etwas interessanter ist dagegen die Beobachtung, dass es in der politischen Sphäre einerseits einen hoch inflationären Gebrauch des Lügenvorwurfs gebe, andererseits sei ein Politiker, der öffentlich einer Lüge überführt werde, in der Regel nicht mehr zu halten. Und diese Konstellation, so suggeriert der Autor, schade dem Image der politischen Klasse insgesamt, weil ihr niemand mehr Glauben schenke.

Diese performative Spreizung des öffentlichen Lügenvorwurfs ist in der Tat markant. Sie ist jedoch insofern nicht weiter überraschend, als diese Spreizung ein Produkt der analysierten Situation ist: Für die jeweilige outgroup ist das gegnerische Image selbst die Lüge, die man beständig zu „entlarven“ sucht, was aber zum Spiel gehört und niemanden sonderlich erregt. Einen veritablen Punkt hingegen gewinnt, wer es schafft, einem Angehörigen der Gegengruppe eine Lüge anzuhängen, die die Eigengruppe des Beschuldigten zur Distanzierung von ihrem Mitglied nötigt, damit das Image der Gruppe nicht zu Schaden kommt. Nur das gilt als performativ „geglückter“ Lügenvorwurf. So viel zum vermeintlichen Sprechakt „Lügen“ (vgl. Falkenberg 1982). Auch die rhetorische Rückzugslinie ist in solchen Fällen klar und verbindlich vorgezeichnet. Sie kommt zum Ausdruck in der FAZ-Überschrift vom 31.7.2013 zur Verantwortlichkeit des Verteidigungsministers in der Euro-HawkAngelegenheit: „De Maizière bestreitet Lüge und gesteht Irrtümer““. 
So kommen wir am Ende doch noch zu einer Revision der soziobiologischen Apologie des Lügens, wie man sie etwa bei Sommer (1993) findet. In der Tat scheint es eine Art evolutionären Rüstungswettlauf zu geben zwischen Strategien der Kommunikation, die sich gegenseitig begrenzen. Besonders moraloide Images liefern in der Hauptsache eine Fassade, hinter der die Spielräume strategischer Nutzenmaximierung ausgeweitet werden können. Das gilt aber paradoxerweise nur, so lange moralische Images die Ausnahme bilden. Sobald sie zur allgemeinen Regel werden, unterminieren sie sich selbst, weil das Image keine Bindewirkung mehr entfaltet. Die Image-Lüge steht darum nicht für eine evolutionär stabile Strategie. Was aber nicht heißt, dass wir sie so schnell loswerden.

\section{Bibliographie}

Alexander, Richard D. (1987): The Biology of Moral Systems. Hawthorne, New York: Aldine.

Bradbury, Jack W. \& Vehrencamp, Sandra L. (2011): Principles of Animal Communication. $2^{\text {nd }}$ ed. Sunderland, Mass.: Sinauer.

Arendt, Hannah (1972): Wahrheit und Lüge in der Politik. Zwei Essays. München: Piper.

BuRKe, Kenneth (1937): Attitudes toward History. $3^{\text {rd }}$ ed. Berkely: University of California Press.

Dawkins, Richard (1976): The Selfish Gene. New York: Oxford UP.

FalKenberG, Gabriel (1982): Lügen. Grundzüge einer Theorie sprachlicher Täuschung. (= Linguistische Arbeiten. 86). Tübingen: Narr.

GIESE, Bettina (1992): Untersuchungen zur sprachlichen Täuschung. (= Reihe germanistische Linguistik. 129). Tübingen: Narr.

GoFFMAN, Erving (1971): Interaktionsrituale. Über Verhalten in direkter Kommunikation. Frankfurt a. M.: SuHRKAMP.

GuSTAFSSON, Lars (1980): Sprache und Lüge. München: Hanser.

KaInZ, Friedrich (1927): „Lügenerscheinungen im Sprachleben“. In: LiPMANN \& Plaut (1927: 212-243).

Lipmann, Otto \& Plaut, Paul (Hrsg.) (1927): Die Lüge in psychologischer, philosophischer, juristischer, pädagogischer, historischer, soziologischer, sprach- und literaturwissenschaftlicher und entwicklungsgeschichtlicher Betrachtung. Leipzig: Ambrosius Barth.

Mauthner, Fritz (1901-02): Beiträge zu einer Kritik der Sprache, 3 Bde. Leipzig (3. Aufl. Leipzig 1923).

Mitschke-Collande, Thomas von (2013): „Radikale Schritte sind nötig“. Süddeutsche Zeitung vom 9./10. November 2013.

MüLLER, Reinhard (2013): „Nichts als die Wahrheit?“. Frankfurter Allgemeine Zeitung vom 31. Juli 2013.

NeCKEL, Sighard (2008): Flucht nach vorn. Die Erfolgskultur der Marktgesellschaft. Frankfurt a. M., New York: Campus.

Schirrmacher, Frank (2013): EGO. Das Spiel des Lebens. München: Karl Blessing.

Sommer, Volker (1993): Lob der Lüge. Täuschung und Selbstbetrug bei Tier und Mensch. 2. Aufl. München: Beck. 
TOMASELLO, Michael (2010): Warum wir kooperieren. Berlin: Suhrkamp.

WeINRICH, Harald (1966): Linguistik der Lüge. Heidelberg: Lambert Schneider.

WiLson, Edward O. (1975): Sociobiology. The New Synthesis. Cambridge, Mass.: Harvard UP.

Zahavi, Amotz \& Zahavi, Avishag (1998): Signale der Verständigung. Das Handicap-Prinzip. Frankfurt a. M.: Insel. 